Article

\title{
Differential Glycemic Effects of Low- versus High-Glycemic Index Mediterranean-Style Eating Patterns in Adults at Risk for Type 2 Diabetes: The MEDGI-Carb Randomized Controlled Trial
}

\author{
Robert E. Bergia ${ }^{1,+}{ }^{-}$, Rosalba Giacco ${ }^{2,3,+}\left(\mathbb{D}\right.$, Therese Hjorth ${ }^{4,+}$, Izabela Biskup ${ }^{4}$, Wenbin Zhu ${ }^{5}$, \\ Giuseppina Costabile $^{2}{ }^{\mathbb{D}}$, Marilena Vitale ${ }^{2} \mathbb{D}$, Wayne W. Campbell ${ }^{1, *}$, Rikard Landberg ${ }^{4}$ and Gabriele Riccardi ${ }^{2} \mathbb{D}$ \\ check for \\ 1 Department of Nutrition Science, Purdue University, 700 West State St., West Lafayette, IN 47907, USA; \\ robbergia@gmail.com \\ 2 Diabetes, Nutrition and Metabolism Unit, Department of Clinical Medicine and Surgery, \\ Federico II University, 80138 Naples, Italy; rgiacco@isa.cnr.it (R.G.); giuseppina.costabile@unina.it (G.C.); \\ marilena.vitale@unina.it (M.V.); gabriele.riccardi@unina.it (G.R.) \\ 3 Institute of Food Science, National Research Council, 83100 Avellino, Italy \\ 4 Department of Biology and Biological Engineering, Food Science and Nutrition, \\ Chalmers University of Technology, 41296 Gothenburg, Sweden; therese.hjorth@chalmers.se (T.H.); \\ izabela.biskup@chalmers.se (I.B.); rikard.landberg@chalmers.se (R.L.) \\ 5 Department of Statistics, Purdue University, West Lafayette, IN 47907, USA; zhu633@purdue.edu \\ * Correspondence: campbellw@purdue.edu \\ + These authors contributed equally to this work.
} updates

Citation: Bergia, R.E.; Giacco, R.; Hjorth, T.; Biskup, I.; Zhu, W.; Costabile, G.; Vitale, M.; Campbell, W.W.; Landberg, R.; Riccardi, G. Differential Glycemic Effects of Lowversus High-Glycemic Index Mediterranean-Style Eating Patterns in Adults at Risk for Type 2 Diabetes: The MEDGI-Carb Randomized Controlled Trial. Nutrients 2022, 14, 706. https://doi.org/10.3390/ nu14030706

Academic Editors: Alessandro Leone and Ramona Silvana De Amicis

Received: 12 December 2021

Accepted: 1 February 2022

Published: 8 February 2022

Publisher's Note: MDPI stays neutral with regard to jurisdictional claims in published maps and institutional affiliations.

Copyright: (c) 2022 by the authors Licensee MDPI, Basel, Switzerland. This article is an open access article distributed under the terms and conditions of the Creative Commons Attribution (CC BY) license (https:// creativecommons.org/licenses/by/ $4.0 /)$

\begin{abstract}
A Mediterranean-style healthy eating pattern (MED-HEP) supports metabolic health, but the utility of including low-glycemic index (GI) foods to minimize postprandial glucose excursions remain unclear. Therefore, we investigated the relative contribution of GI towards improvements in postprandial glycemia and glycemic variability after adopting a MED-HEP. We conducted a randomized, controlled dietary intervention, comparing high- versus low-GI diets in a multi-national (Italy, Sweden, and the United States) sample of adults at risk for type 2 diabetes. For 12 weeks, participants consumed either a low-GI or high-GI MED-HEP. We assessed postprandial plasma glucose and insulin responses to high- or low-GI meals, and daily glycemic variability via continuous glucose monitoring at baseline and post-intervention. One hundred sixty adults (86 females, 74 males; aged $55 \pm 11 \mathrm{y}$, BMI $31 \pm 3 \mathrm{~kg} / \mathrm{m}^{2}$, mean $\pm \mathrm{SD}$ ) with $\geq$ two metabolic syndrome traits completed the intervention. Postprandial insulin concentrations were greater after the high-GI versus the low-GI test meals at baseline $(p=0.004)$, but not post-intervention $(p=0.17)$. Postprandial glucose after the high-GI test meal increased post-intervention, being significantly higher than that after the low-GI test meal $(35 \%, p<0.001)$. Average daily glucose concentrations decreased in both groups post-intervention. Indices of 24-h glycemic variability were reduced in the low-GI group as compared to baseline and the high-GI intervention group. These findings suggest that low-GI foods may be an important feature within a MED-HEP.
\end{abstract}

Keywords: Mediterranean diet; metabolic syndrome; metabolic health; impaired glycemic control; metabolic risk factors; insulinemia; glycemic variability; continuous glucose monitoring; oral glucose tolerance test; meal glucose tolerance test

\section{Introduction}

Type 2 diabetes is a dire metabolic condition that has a profound impact on the estimated $\sim 400$ million individuals afflicted worldwide [1]. Without a rapid and robust response, type 2 diabetes is projected to continue along this course of precipitous increases in cases during the upcoming decades [1,2]. The deleterious effects of type 2 diabetes are further magnified when its contributions towards cardiovascular disease, the leading cause of mortality in Western nations, are considered [3,4]. Given the societal burden, research 
investigating the potential for lifestyle and dietary interventions for those who are at risk of developing type 2 diabetes, must be fully leveraged if there is to be hope in slowing the steep rise in cases [5].

Postprandial glycemia may contribute as much or more than fasting blood glucose to the pathogenesis of impaired insulin sensitivity and insulin secretion seen in the progression towards type 2 diabetes [6,7]. Indeed, the loss of postprandial glucose control often manifests before derangements in fasting indices, along the path towards type 2 diabetes in at-risk individuals [8]. Therefore, strategies to attenuate postprandial glucose excursions may be of particular importance in the effort to reduce the global burden of disease. Proposed 40 years ago, the glycemic index (GI) of carbohydrate-containing foods is posited to play a substantial role in postprandial glucose excursions [9]. However, there is still not a consensus on the relevance and utility of GI in non-diabetic people [10], particularly in the context of a healthy eating pattern (HEP) where other health-promoting dietary factors may take precedence [11]. It is unclear whether differences in postprandial glucose and insulin responses that are induced acutely by various carbohydrate foods may disappear after a few weeks due to compensatory mechanisms operative in non-diabetic people [12].

Consuming a Mediterranean-style (MED) HEP is supported by considerable evidence suggestive of a reduced risk of type 2 diabetes development $[13,14]$. The preponderance of the evidence is observational in nature, with relatively little experimental evidence, particularly outside of the geographical Mediterranean region, supporting this HEP. Fat quality, dietary fiber, and different bioactive compounds have been emphasized as the core elements behind the observed health effects of a MED-HEP [15]. However, to the best of our knowledge, there have been no studies assessing the relative contributions of highversus low-GI foods to improvements in indices of glucose control and cardiometabolic health in the context of a MED-HEP.

Therefore, we conducted a 12-week, multi-center, randomized, controlled intervention assessing the potential differential effects of iso-caloric, weight-maintaining, high-versus low-GI MED-HEPs on indices of glucose control among participants at risk for developing type 2 diabetes. We hypothesized that the low-GI group would present with lower postprandial glucose and insulin responses relative to the high-GI group at baseline and that these differential responses would be maintained at the end of the dietary intervention. Further, we hypothesized that the low-GI group would present with improvement in indices of glycemic variability from baseline to post-intervention, relative to the high-GI group.

\section{Materials and Methods}

The MEDGI-Carb trial is an international multi-center randomized, controlled, parallelgroup, 15-week trial including a 3-week baseline period followed by 12 weeks of controlled dietary intervention. This research study was initiated in January 2018 and the trial continued through December 2019. This study was conducted at three centers: (1) Federico II University, Naples, Italy (2) Chalmers University of Technology, Gothenburg, Sweden, and (3) Purdue University, West Lafayette, IN, USA. The study protocol was approved by the institutional review boards at Purdue University and Federico II University and by the Swedish Ethical Review Authority. This study was registered in the public trial registry Clinicaltrials.gov (accessed on 7 December 2021) as NCT03410719. Detailed descriptions of study protocols for all centers are published [16].

\subsection{Experimental Design}

During the 12-week intervention period, subjects consumed a controlled, iso-energetic, weight-maintenance diet and were instructed to consume intervention-specific foods to achieve a low-glycemic or high-glycemic MED-HEP (low-GI or high-GI, respectively). Outcome measurements were obtained on standardized testing days to determine markers of glucose homeostasis by completion of an eight-hour meal glucose tolerance test (MGTT), including both breakfast and lunch resembling food choices of the assigned diet, an oral glucose tolerance test (OGTT), and 24-h continuous glucose monitoring on separate days 
at baseline and post-testing. Primary outcomes are postprandial plasma glucose and insulin responses during the MGTT; secondary outcomes include fasting and OGTT plasma glucose and insulin; blood $\mathrm{HbA}_{1 \mathrm{c}}$; and indices of 24-h glycemic variability.

\subsection{Eligibility Criteria}

The eligibility criteria were designed to select middle-aged and older adults at risk for developing type 2 diabetes. Therefore, adults with a waist circumference $>102 \mathrm{~cm}$ (males) or $>88 \mathrm{~cm}$ (females) and one additional trait of the metabolic syndrome, according to the National Cholesterol Education Program's Adult Treatment Panel III [17], were recruited. The additional traits could include blood pressure $>130 / 85 \mathrm{mmHg}$ or taking medication to control high blood pressure; fasting plasma glucose 5.6-7.0 mmol/L; fasting triglycerides $1.7-4.5 \mathrm{mmol} / \mathrm{L} ; \mathrm{HDL}<1.0 \mathrm{mmol} / \mathrm{L}$ (males) or $<1.3 \mathrm{mmol} / \mathrm{L}$ (females). A member of the research team at each of the three testing sites, who was not involved in data collection or analysis, generated the random allocation sequence and assigned subjects to the interventions. Each subject was randomly assigned to one of two dietary groups using either a stratified block pattern (Italy \& US; $n=8,10$ blocks; 4 randomized to each group per block of 8 , using an online randomization plan generator (http:/ / randomization. com/ (accessed on 31 January 2022) or a mixed size of the block pattern (Sweden; 4, 6, and 8 subjects per block in random block order, using Rstudio software version 2.4.0 (RStudio, Boston MA, USA) with package 'blockrand' version 1.3)). The randomization code remained unrevealed until all participant testing and analyses of samples for a priori primary outcomes were completed. Full inclusion criteria details, recruitment, and consent procedures can be found elsewhere [16].

\subsection{Dietary Control}

During the 3-week baseline period, all subjects consumed their habitual, self-chosen, unrestricted diets. Throughout the 12-week intervention period, each subject was counseled to follow their assigned iso-caloric MED-HEP using a combination of prescribed menus (breakfast, lunch, and snacks) and an item-specific version of the 'Dinner Recipe Builder' for dinner. The Dinner Recipe Builder is a mechanism to self-efficacy by which participants were given the flexibility to mix and match ingredients, while still following a MED-HEP. The two group-specific diet plans contained primarily the same foods and beverages in their MED-HEPs, except for substitutions of major sources of starch in the meals. All participants were advised to consume the same quantity of metabolizable carbohydrate $(270 \mathrm{~g} / \mathrm{d})$ and fiber $(35 \mathrm{~g} / \mathrm{d})$. Higher or lower energy content was achieved through the modulation of dietary fat and protein. One-half of daily carbohydrate (135 g) was the same for both the low- and high-GI groups, including the carbohydrates in fruits, vegetables, and other foods that all subjects consumed. The other one-half of the daily carbohydrate intake (135 g) was specific to the low-GI and high-GI groups. Specifically, $135 \mathrm{~g}$ of carbohydrate in the low-GI group came from foods with GI values $<55$, while $135 \mathrm{~g}$ of carbohydrate in the high-GI group came from foods with GI values $>70$. These GI cut-points correspond with those indicative of low-GI foods $(<55)$ and high-GI foods $(>70)$ [18]. The interventionspecific carbohydrates were distributed as $35 \mathrm{~g}$ at breakfast, $40 \mathrm{~g}$ at lunch, and $60 \mathrm{~g}$ at dinner. Complete descriptions of dietary controls can be found elsewhere [16]. Briefly, all participants were provided with group-specific instructions on the quantities of specific foods to consume. They were also given selected food items to use for their meals (high-GI jasmine rice, potato, mashed potatoes, couscous, wholegrain bread, and rusks; low-GI pasta, brown rice, flatbread, all bran, and wheat, plus rye bread and seeds). Dietary counseling was given bi-weekly and included group dinner meal preparation sessions.

\subsection{Postprandial Assessments}

Visits for clinical assessments at baseline and post-intervention included an 8-h MGTT and a 2-h OGTT (Supplemental Figure S1). Prior to all testing days, participants were instructed not to eat or drink anything (except a small amount of water) from 22:00 $\mathrm{h}$ the 
evening before the visit. Participants were counseled to refrain from vigorous physical activity for $48 \mathrm{~h}$ prior to testing days, avoid alcohol $24 \mathrm{~h}$ prior to testing days, and avoid caffeinated beverages the morning of testing days. After arriving at the testing facilities, participants would be seated in a chair/bed to rest. A catheter was placed in an antecubital vein and remained in place for the remainder of the testing day. Blood pressures were taken in duplicate after 15 min of rest.

For the OGTT and MGTT, double baseline fasting blood samples were collected at the minus 15-min time-point and the minus 5-min time-point following 15 min of rest (pooled ((mean value)) and denoted as time-point -10). The $75 \mathrm{~g}$ glucose drink (OGTT) or test meal (MGTT) were consumed at time-point 0. Full test meal contents are provided in Supplemental Table S1. Each participant consumed a test meal consistent with the composition of the assigned diet, at baseline, and at the end of the intervention. During the OGTT, blood samples were collected at $60 \mathrm{~min}$ and $120 \mathrm{~min}$ after consumption of the test glucose beverage. Subjects were not permitted additional fluid consumption during the test. During the MGTT, blood samples were collected immediately following the test breakfast meal (time-point +15 ) and then at intervals progressing from $15 \mathrm{~min}$ to one hour from time-points $+30,45,60,90,120,180$, and 240 . The second test meal was provided following the time-point +240 blood draw. The blood draw pattern of timing was repeated after the second meal was consumed.

\subsection{Continuous Glucose Monitoring}

Medtronic iPro2 Professional continuous glucose monitoring devices (Northridge, CA, USA) were used to obtain 24-h interstitial glucose concentrations in 5-min intervals during the baseline and post-testing weeks. Data were entered into the EasyGV platform (University of Oxford, Oxford, England) for calculation of the relevant indices of glycemic variability in non-diabetic individuals (mean amplitude of glucose excursions; continuous overall net glycemic action; mean absolute glucose; and lability index).

\subsection{Blood Collection and Analysis}

During the 12-week intervention period, subjects consumed a controlled, iso-energetic, weight-maintenance diet and were instructed to consume intervention-specific foods to achieve a low-glycemic or high-glycemic MED-HEP (low-GI or high-GI, respectively). Complete descriptions of blood sample collection analyses can be found elsewhere [16]. Briefly, blood samples were obtained from an antecubital vein and placed in tubes containing a clot activator to obtain serum or sodium/lithium heparin to obtain plasma. Serum tubes were held at room temperature for at least $15 \mathrm{~min}$ and then centrifuged at $4000 \times g$ at $4{ }^{\circ} \mathrm{C}$ for $15 \mathrm{~min}\left(3000 \times \mathrm{g}\right.$ at $4^{\circ} \mathrm{C}$ for $10 \mathrm{~min}$ in Sweden). EDTA plasma, serum, and heparinized plasma samples were immediately refrigerated/kept on ice, processed, and aliquoted into microtubes. Plasma and serum aliquots were frozen at $-20^{\circ} \mathrm{C}$ within $2 \mathrm{~h}$ of sample collection, stored at this temperature for a maximum of one week, and then stored at $-80^{\circ} \mathrm{C}$ until thawed for analysis. EDTA plasma samples were used to assess insulin and glucose concentrations. All samples were analyzed at the end of the study to minimize batch effects.

\subsection{Statistical Analyses}

The primary analyses followed the intention-to-treat plan. A repeated measures linear mixed model was used to model the main effects of group, time, and group $\times$ time interactions as well as the effect of the study center. Least square means for outcomes of interest were calculated via the LSMESTIMATE statement using the PROC MIXED procedure in SAS statistical software version 9.4 (SAS Institute, Cary, NC, USA). Power calculations indicated that 180 subjects total (90 low-GI, 90 high-GI; 60 from each testing center) would provide greater than $80 \%$ power to detect a $30 \%$ differential response between the dietary interventions for the primary endpoint (postprandial insulin) with similar variation reported in the study by Giacco et al. [19]. Postprandial glucose and insulin 
averages were assessed by setting the minimum-reported value of the participant as the baseline, whereby all subsequent values were firstly subtracted from the minimum values, and then were averaged. The same standard was used in the calculation of postprandial glucose and insulin areas under the curve (AUC, using the trapezoidal rule). This method minimizes the conceptual errors associated with large glucose excursions washing out and not being represented in postprandial assessments, i.e., high glycemic variability where large peaks (hyperglycemia) are followed by large depressions below fasting concentrations (hypoglycemia). Calculation with the $\mathrm{AUC}_{\min }$ method consistently resulted in the lowest intra- and inter-individual coefficients of variation in previous assessments of postprandial glycemia [20].

When confronted with missing data from postprandial assessments, multiple imputation using chained equation procedures were followed in accordance with Rubin's rules [21] to combine the statistical results from each individual imputed complete data. Covariates accounted for in all statistical models included BMI, waist circumference, age, sex, smoking status (smoker/non-smoker), and testing center (Italy, Sweden, or USA). When no significant group $\times$ time interaction was observed, data from both groups were pooled to assess the overall effect of the dietary intervention. Significance was set at $p<0.05$.

\section{Results}

The CONSORT participant flow diagram is presented in Figure 1. During the clinical testing phase (February 2018 to December 2019), 584 participants were screened for eligibility. Of the initially enrolled 213 participants, 27 (low-GI; $n=12$, high-GI; $n=15$ ) dropped out prior to commencing the dietary intervention, and 26 (low-GI; $n=8$, high-GI; $n=18$ ) dropped out during the dietary intervention, resulting in 160 (low-GI; $n=86$, high-GI; $n=74$ ) participants completing the dietary intervention.

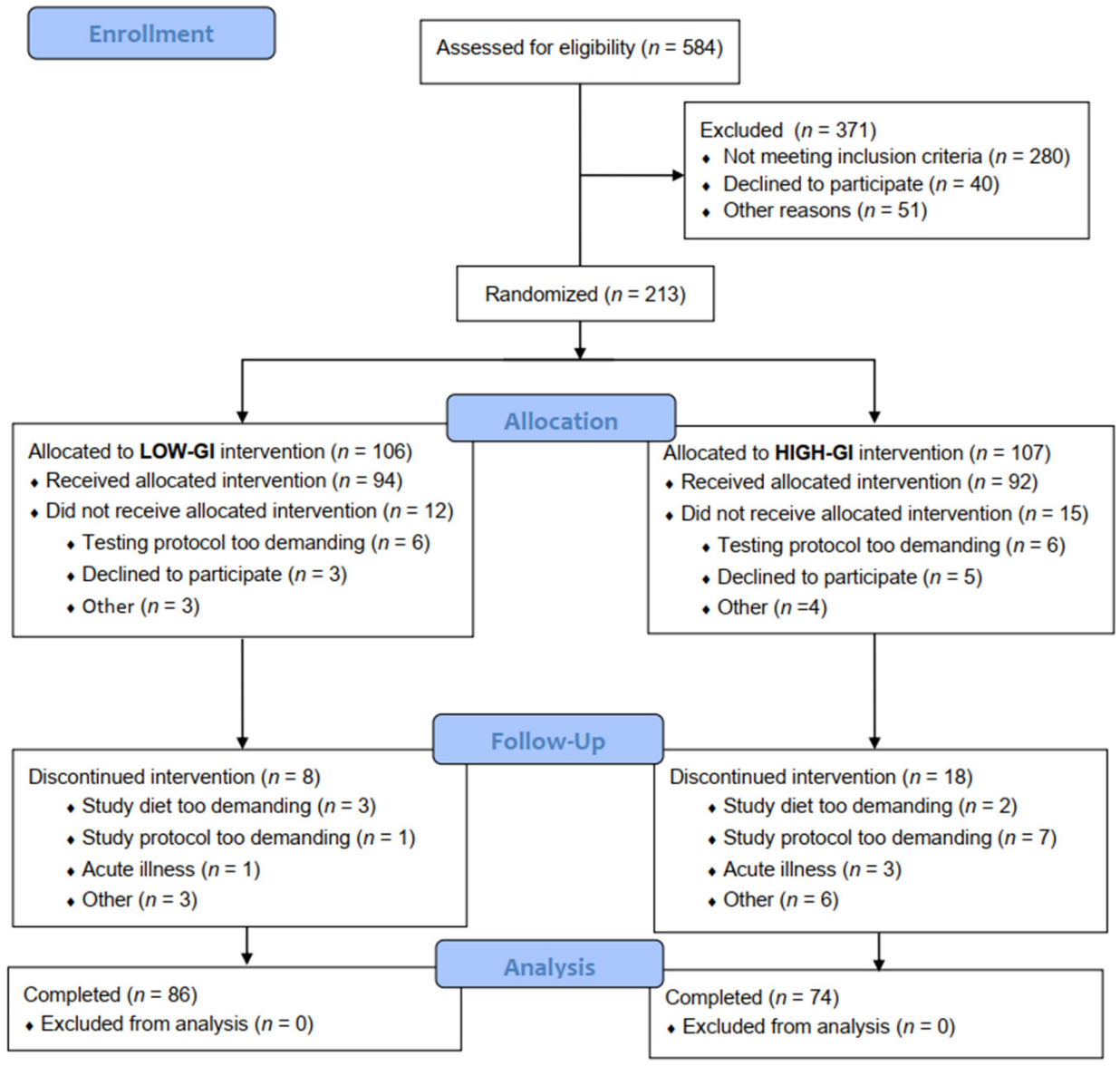

Figure 1. CONSORT participant flow diagram. 


\subsection{Baseline Characteristics}

There were 119 female participants and 94 male participants (Table 1). In our sample of adults at risk for type 2 diabetes, all participants presented with elevated waist circumference (by design). Forty-four percent of participants had two traits of the metabolic syndrome, 37\% had three traits (and thus are classified as having the metabolic syndrome), $16 \%$ presented with four traits, and $3 \%$ had all five traits. Elevated blood pressure was the most common secondary trait (60\%), followed by elevated fasting glucose $(46 \%)$, low HDL $(38 \%)$, and elevated triglycerides (33\%).

Table 1. Fasting clinical characteristics of participants at screening.

\begin{tabular}{lcc}
\hline Demographic Characteristics & $\begin{array}{c}\text { Low-GI } \\
(\boldsymbol{n}=\mathbf{1 0 2})\end{array}$ & $\begin{array}{c}\text { High-GI } \\
(\boldsymbol{n}=\mathbf{1 1 1})\end{array}$ \\
\hline Age at randomization (years) & $55 \pm 10$ & $55 \pm 11$ \\
\hline Female $n(\%)$ & $55(53.9 \%)$ & $64(57.7 \%)$ \\
\hline Weight $(\mathrm{kg})$ & $92 \pm 14$ & $88 \pm 14$ \\
\hline BMI $\left(\mathrm{kg} / \mathrm{m}^{2}\right)$ & $31.1 \pm 3.1$ & $30.3 \pm 3.0$ \\
\hline Waist Circumference $(\mathrm{cm})$ & $106 \pm 8$ & $105 \pm 9$ \\
\hline Metabolic characteristics & & $5.4 \pm 0.7$ \\
\hline Glucose $(\mathrm{mmol} / \mathrm{L})$ & $5.4 \pm 0.6$ & $5.0 \pm 0.9$ \\
\hline Total cholesterol $(\mathrm{mmol} / \mathrm{L})$ & $4.9 \pm 1.0$ & $1.3 \pm 0.6$ \\
\hline Triglycerides $(\mathrm{mmol} / \mathrm{L})$ & $1.3 \pm 0.6$ & $1.3 \pm 0.4$ \\
\hline HDL $(\mathrm{mmol} / \mathrm{L})$ & $1.3 \pm 0.4$ & $3.3 \pm 0.7$ \\
\hline LDL $(\mathrm{mmol} / \mathrm{L})$ & $3.2 \pm 0.8$ & $126 \pm 14$ \\
\hline Systolic blood pressure $(\mathrm{mm} \mathrm{Hg})$ & $126 \pm 14$ & $83 \pm 8$ \\
\hline Diastolic blood pressure $(\mathrm{mm} \mathrm{Hg})$ & $82 \pm 9$ & \\
\hline
\end{tabular}

Data are means \pm SD. There were no statistically significant differences at baseline.

\subsection{Dietary Composition}

At baseline, the two groups did not present with differences in any dietary features (Supplemental Table S2). Post-intervention, both groups reduced their intakes of total saturated and polyunsaturated fat, while their intakes of fiber, monounsaturated fat, and carbohydrates increased. Post-intervention there was no difference in energy or nutrient composition between the low- and high-GI groups. Targeted differences in the glycemic index were achieved with average glycemic index values of $46.8 \pm 3.1$ vs. $66.2 \pm 4.7$ reported in the low- and high-GI groups, respectively.

\subsection{Postprandial MGTT Glucose and Insulin Responses}

Postprandial insulin and glucose responses to the 8-h MGTT are presented in Figure 2. Average postprandial insulin was greater after the high-GI test meals compared to the Low-GI test meals at baseline $(p=0.004)$, but this difference was no longer present postintervention $(p=0.17)$. From baseline to post-intervention, postprandial insulin decreased in the high-GI group, but not in the low-GI group (group $\times$ time; $\Delta-30.6 \pm 15.3 \mathrm{pmol} / \mathrm{L}$; $p=0.046)$. Postprandial glucose was greater $(\sim 17 \%)$ after the high-GI vs. the Low-GI test meals at baseline $(p=0.02)$, and more so post-intervention $(35 \% ; p<0.001)$. This greater difference between low- and high-GI groups post-intervention was attributable to increases in average postprandial glucose over the 12 weeks in the high-GI group $(\Delta 0.2 \pm 0.1 \mathrm{mmol} / \mathrm{L} ; p=0.03)$. Similar to postprandial insulin responses, glucose responses in the low-GI group were unchanged after the intervention. Overall, there were more robust differential effects of GI between the groups at the lunch meals, while glucose and insulin responses were less different after the breakfast meals (Supplemental Figure S2). Postpran- 
dial glucose and insulin AUC results were comparable to those seen for postprandial averages (Supplemental Figure S3).

Our study was not designed to determine differences in glycemic responses based on sex or race/ethnicity per se. Most participants were Caucasian non-Hispanic $(n=158,99 \%)$, therefore, no meaningful post hoc analyses can be conducted. Sex effects were detected during modeling postprandial insulin, but not postprandial glucose responses.
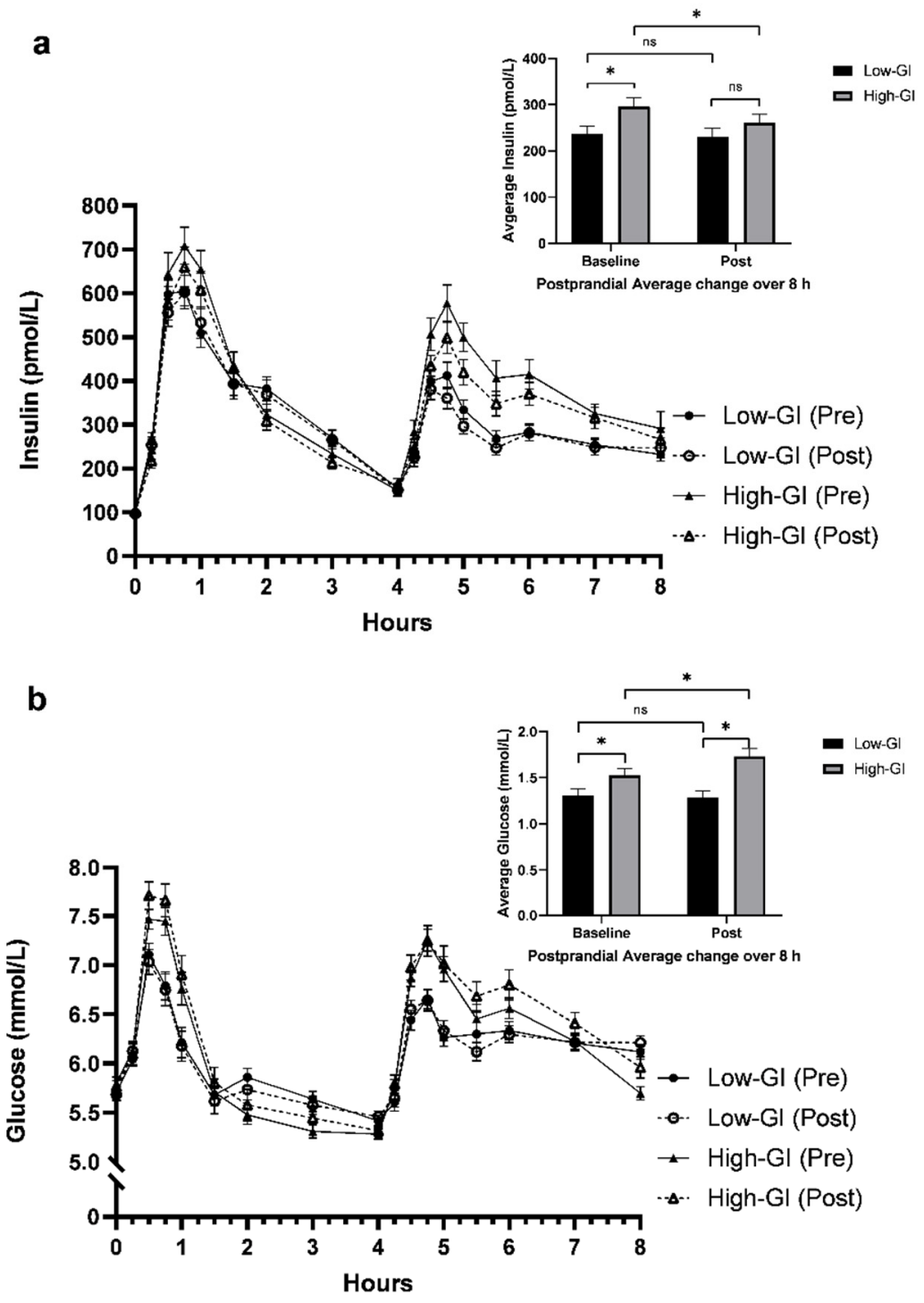

Figure 2. Insulin (a) and glucose (b) responses to a low-GI and high-GI 8-h meal glucose tolerance test at baseline and after a 12-week dietary intervention. Inset bar graphs display average postprandial insulin and glucose elevations above fasting concentrations over the 8-h period. Data are means \pm SEM. * Statistically significant, $p<0.05$. ns, no significance.

\subsection{4-h Glycemic Variability}

At baseline, all 24-h continuous glucose monitoring variables were not different between the groups (Figure 3). Average 24-h glucose concentrations decreased in both groups, with indices of glycemic variability improving in the low-GI group, but not in the high-GI group. The low-GI group presented with reductions in 24-h standard deviation, mean amplitude of glucose excursions, and mean absolute glucose. Lability index $(p=0.04)$, mean amplitude of glucose excursions $(p<0.01)$, and mean absolute glucose $(p=0.02)$ were lower in the low-GI group compared to the high-GI group post-intervention. 


\section{a}
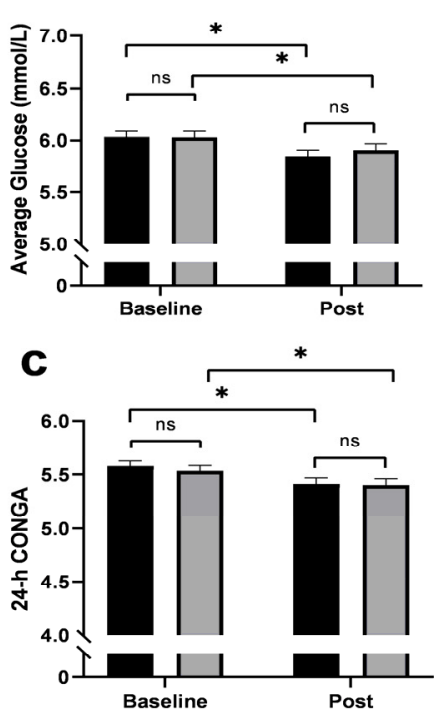

e

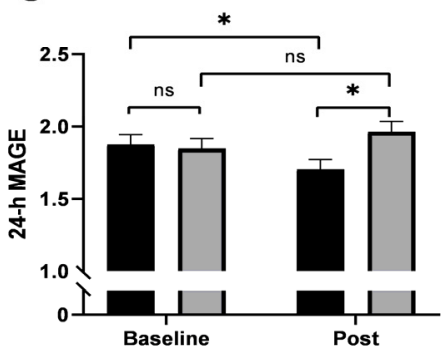

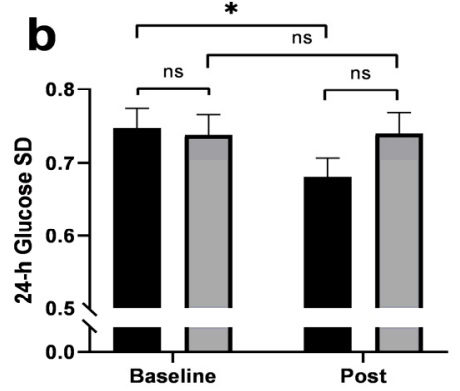

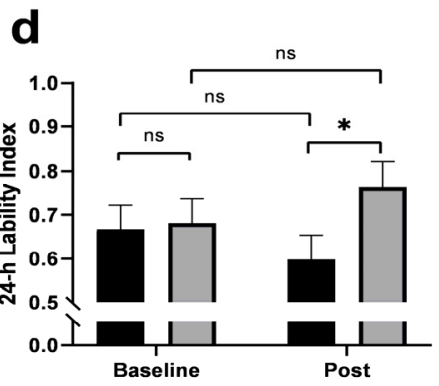

$\mathbf{f}$

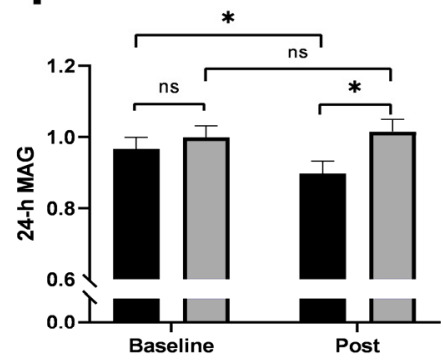

Figure 3. 24-h continuous glucose monitor-derived measures of glycemic variability at baseline and after a 12-week dietary intervention. (a) Average 24-h glucose concentration, (b) standard deviation (SD), (c) continuous overall net glycemic action (CONGA), (d) lability index, (e) mean amplitude of glucose excursions (MAGE), and (f) mean absolute glucose (MAG). Presented data are means \pm SEM. * Statistically significant, $p<0.05$. ns, no significance.

\subsection{Fasting and OGTT Glucose and Insulin Responses}

There were no differences in 1-h or 2-h glucose (Supplemental Figure S4a) and insulin (Supplemental Figure S4b) between the low- and high-GI groups at either baseline or post-intervention. Irrespective of GI, glucose responses, but not insulin, decreased over the course of the intervention (pooled analysis; $p=0.02$ ). The GI of the MED-HEP did not influence changes over time in the fasting risk factors for type 2 diabetes, including fasting glucose, insulin, HOMA-IR, and $\mathrm{HbA}_{1 \mathrm{c}}$ (Supplemental Table S3).

\section{Discussion}

This study demonstrated that consuming a Mediterranean-style eating pattern with low-GI foods reduces daily glycemia and indices of glycemic variability in adults at risk for type 2 diabetes. As hypothesized, postprandial glycemia was lower after the lowGI test meals relative to the high-GI test meals at baseline, and the magnitude of this difference increased during the dietary intervention. This widened difference was primarily attributable to increases in the high-GI group's postprandial glucose, while the low-GI group's glucose responses were not different between baseline and post-intervention. This suggests that a diet based on high-GI foods, including $50 \%$ of carbohydrates from foods with GI values $>70$, significantly increases the postprandial glucose response, not only in an acute setting but also in the long term despite adherence to an overall healthy eating pattern. 
Contrary to our hypothesis, while postprandial insulin was lower in the low-GI group compared to the high-GI group at baseline, this difference was no longer present post-intervention; in fact, postprandial insulin decreased at post-intervention more in the high-GI group relative to the low-GI group. However, this decline in the postprandial insulin occurred only at breakfast, while plasma insulin concentrations after lunch remained significantly higher during the intervention in the high-GI group. Indeed, the difference in postprandial insulin at lunch was more than $50 \%$ higher in the high-GI group. Postprandial plasma insulin concentrations are generally considered a marker of increased cardiovascular risk and therefore the increased postprandial insulin concentrations after lunch might be considered as a potential untoward effect of the high GI diet [22].

In contrast to the clear benefits of a low-GI diet over a high-GI diet regarding its effects on postprandial glucose metabolism, we detected no improvement in fasting glucose, OGTT, or $\mathrm{HbA}_{1 c}$. This was unsurprising, as fasting plasma glucose concentrations are typically unaffected by weight-maintaining dietary interventions, particularly when they are not clearly elevated [23]. In addition, $\mathrm{HbA} 1 \mathrm{c}$ is an imprecise marker of glucose control for values below $5.5 \%(37 \mathrm{mmol} / \mathrm{mol})$, which is the range in which most of our participants were included [24]. Regarding OGTT responses, improvements in this marker are only reported in long-term weight-loss interventions in individuals with diagnosed impaired glucose tolerance [25].

Our study results are concordant with the outcomes of one previous study [26] where individuals with prediabetes displayed improved postprandial glucose control in response to a low-GI diet, but they are not completely concordant with the OmniCarb trial, a similar intervention involving manipulation of GI within a dietary intervention in which plasma insulin concentrations were not influenced by GI [11]. While low-GI diets have clinical utility in individuals with type 2 diabetes [27], our findings support the hypothesis that replacing high-GI foods for low-GI foods might also be useful for improving indices of glycemic control in people with traits of metabolic syndrome, thereby potentially reducing the risk of progression to type 2 diabetes and/or cardiovascular disease [28].

The results from the 24-h continuous glucose monitoring add important context to our postprandial findings. We report that while the mean 24-h glucose concentrations decreased comparatively in both groups following MED-HEPs, changes in the indices of glycemic variability favored the low-GI group. While there is currently a limited consensus on the relative importance of different indices of glycemic variability [29], the consistent improvement observed in the low-GI group across classical and contemporary indices strongly suggests a more stable blood glucose profile after the low-GI diet. The classical indices include the mean amplitude of glucose excursions, mean absolute glucose, and standard deviation, while the contemporary indices include lability index and continuous overall net glycemic action. Mechanistic support for the relevance of glycemic variability can be found in research demonstrating that acute glucose excursions induce oxidative stress to a greater degree than sustained hyperglycemia [6]. However, the clinical relevance of reducing blood glucose excursions remains unresolved, with some [30], but not all [31] evidence supporting glycemic variability as an independent risk factor for overall mortality and cardiovascular diseases. Assessment of glucose variability in individuals at risk for type 2 diabetes, such as our participants, may be particularly important as perturbations in glucose homeostasis may be an early manifestation of glucose dysregulation [32].

We chose to assess the effects of GI in the context of a MED-HEP because the MED-HEP has been linked with a reduced progression to type 2 diabetes in at-risk populations [33]. Indeed, the MED-HEP appears to be particularly effective in improving glycemic control relative to other dietary patterns such as DASH which may be comparatively more effective in reducing blood pressure [34]. Components of the MED-HEP, such as abundant monounsaturated fatty acids in olive oil, may also particularly improve postprandial glycemia via an improved postprandial insulin sensitivity [35].

Strengths of the MEDGI-Carb study include strong clinical design features such as randomization, blinding of the analytical procedures, and an appropriate sample size with 
respect to effect size and power based on previous research. Further, the robustness of the primary manipulated variable (GI of the carbohydrate foods) was ensured through independent analyses to ensure that the GI of the carbohydrate foods were sufficiently different between the low- and high-GI groups, which had been intended but was difficult to achieve in previous studies. A potential limitation stems from our study sample consisting almost entirely of participants of Caucasian ethnicity. Certain populations, such as Asian individuals, display unique metabolic characteristics that may predispose them to benefit more from the interventions posited to influence the postprandial carbohydrate metabolism [36]. Therefore, care should be taken that these findings are not overgeneralized to people from other racial groups. In addition, our study was tailored to assess the metabolic effects of the dietary intervention but it was not powered for a duration and sample size that would allow for the evaluation of the impact of the diets on clinical events. Lastly, we cannot discount that the health-promoting effects of GI reduction may be greater in the context of a higher-carbohydrate pattern, such as a typical 'dietary approaches to stop hypertension' HEP [37], compared with a lower-carbohydrate diet, such as a MED-HEP [38]. Therefore, future studies should investigate both the potential modulation of postprandial glycemia by the monounsaturated fats commonly found in MED-HEPs and assess the potential GI-induced differences from MED-HEP varieties featuring a higher percentage of daily energy from carbohydrates.

\section{Conclusions}

In conclusion, the acute superiority in the indices of postprandial glucose control of participants who emphasized low-GI foods relative to those who emphasized high-GI foods is sustained and was further amplified over time in the context of a background MED-HEP. However, this amplified difference over time is primarily due to a worsening of postprandial glucose control among participants whose diets emphasized high-GI foods. Unexpectedly, the higher insulin responses to consuming high-GI versus low-GI meals at baseline were not apparent after adopting the MED-HEP. Consuming a MED-HEP was sufficient to reduce daily glycemia, but only the low-GI diet resulted in improvements in the indices of daily glycemic variability. Collectively, these findings demonstrate the relevancy of GI within a mixed diet with healthy features resembling those of a traditional Mediterranean-style diet among non-diabetic individuals. Since low-GI foods are an inherent element of a traditional Mediterranean diet [39], our findings suggest that low-GI foods may contribute to the health benefits seen from the MED-HEP.

Supplementary Materials: The following supporting information can be downloaded at: https: / / www.mdpi.com/article/10.3390/nu14030706/s1, Figure S1: Meal- and oral glucose tolerance testing day schematic, Figure S2: Insulin and glucose elevations above fasting concentrations in response to low-GI and high-GI Breakfast and Lunch Meals During 8-h Meal Glucose Tolerance Tests at Baseline and After a 12-week Dietary Intervention, Figure S3: Insulin and Glucose Postprandial Areas Under the Curve in Response to low-GI and high-GI Meals During 8-h Meal Glucose Tolerance Tests at Baseline and After a 12-week Dietary Intervention, Figure S4: 1-h and 2-h glucose and insulin responses at to a 2-h oral glucose tolerance test (75 g glucose bolus), Table S1: Food Composition of Standardized Meal Glucose Tolerance Test Meals, Table S2: Dietary Composition Before And After A low- And high-GI Mediterranean Dietary Intervention, Table S3: Effect of low- versus high-GI Healthy Eating Patterns on Risk Factors for Type 2 Diabetes.

Author Contributions: Conceptualization, R.E.B., R.L., G.R. and W.W.C.; methodology, R.E.B., W.W.C., I.B., R.L. and G.R.; formal analysis, W.Z.; investigation, R.E.B., R.G., I.B., G.C. and M.V.; resources, W.W.C., R.L. and G.R.; data curation, W.Z.; writing-original draft preparation, R.E.B.; writing-review and editing, R.E.B., R.G., W.W.C., T.H., I.B., W.Z., G.C., R.L. and G.R.; supervision, G.R., R.L. and W.W.C.; project administration, R.E.B., R.G., I.B., G.C. and M.V.; funding acquisition, G.R., R.L. and W.W.C. All authors have read and agreed to the published version of the manuscript. 
Funding: This research was funded by Barilla International and Barilla USA. The funding sources had no role in the collection, analysis, and interpretation of data; in writing of this and any reports; and in the decision to submit the article for publication.

Institutional Review Board Statement: The study was conducted according to the guidelines of the Declaration of Helsinki, and was approved by the institutional review boards at Purdue University and Federico II University and by the Swedish Ethical Review Authority. This study was registered in the public trial registry Clinicaltrials.gov (accessed on 7 December 2021) as NCT03410719.

Informed Consent Statement: Informed consent was obtained from all subjects involved in the study.

Data Availability Statement: The data presented in this study are available on request from the corresponding author.

Acknowledgments: W.W.C., G.R. and R.L. served as co-principal investigators and are thus co-senior authors. The study authors would like to thank all study participants who volunteered their valuable time and attention. We thank Barilla G\&R F.lli. SpA, Parma, Italy for providing some of the cereal products for the study participants.

Conflicts of Interest: G.R. is a member of the Scientific Advisory Board of the Nutrition Foundation of Italy, the Barilla Foundation for Food and Nutrition, and the Istituto Nutrizionale Carapelli Foundation; he is a member of the Health and Wellbeing Advisory Board of the Barilla G. e R. Fratelli Company. R.B. is currently employed by ADM. Research presented in this paper was conducted in a former role and has no connection with ADM. I.B. is currently employed by AstraZeneca. Research presented in this paper was conducted in a former role and has no connection with AstraZeneca.

\section{References}

1. Saeedi, P.; Petersohn, I.; Salpea, P.; Malanda, B.; Karuranga, S.; Unwin, N.; Colagiuri, S.; Guariguata, L.; Motala, A.A.; Ogurtsova, K.; et al. Global and regional diabetes prevalence estimates for 2019 and projections for 2030 and 2045: Results from the International Diabetes Federation Diabetes Atlas, 9th edition. Diabetes Res. Clin. Pract. 2019, 157, 107843. [CrossRef] [PubMed]

2. Kaiser, A.B.; Zhang, N.; Der Pluijm, W.V. Global prevalence of type 2 diabetes over the next ten years (2018-2028). Diabetes 2018, 67 (Suppl. 1), 202-LB. [CrossRef]

3. Dokken, B.B. The pathophysiology of cardiovascular disease and diabetes: Beyond blood pressure and lipids. Diabetes Spec. 2008, 21, 160-165. [CrossRef]

4. Xu, J.; Murphy, S.L.; Kockanek, K.D.; Arias, E. Mortality in the United States, 2018. NCHS Data Brief. 2020, 355, 1-8. [PubMed]

5. Yamaoka, K.; Tango, T. Efficacy of lifestyle education to prevent type 2 diabetes: A meta-analysis of randomized controlled trials. Diabetes Care. 2005, 28, 2780-2786. [CrossRef] [PubMed]

6. Monnier, L.; Lapinski, H.; Colette, C. Contributions of fasting and postprandial plasma glucose increments to the overall diurnal hyperglycemia of type 2 diabetic patients: Variations with increasing levels of HbA1c. Diabetes Care 2003, 26, 881-885. [CrossRef]

7. Blaak, E.E.; Antoine, J.M.; Benton, D.; Bjorck, I.; Bozzetto, L.; Brouns, F.; Diamant, M.; Dye, L.; Hulshof, T.; Holst, J.J.; et al. Impact of postprandial glycaemia on health and prevention of disease. Obes. Rev. 2012, 13, 923-984. [CrossRef]

8. Monnier, L.; Colette, C.; Dunseath, G.J.; Owens, D.R. The loss of postprandial glycemic control precedes stepwise deterioration of fasting with worsening diabetes. Diabetes Care 2007, 30, 263-269. [CrossRef] [PubMed]

9. Jenkins, D.J.; Wolever, T.M.; Taylor, R.H.; Barker, H.; Fielden, H.; Baldwin, J.M.; Bowling, A.C.; Newman, H.C.; Jenkins, A.L.; Goff, D.V. Glycemic index of foods: A physiological basis for carbohydrate exchange. Am. J. Clin. Nutr. 1981, 34, 362-366. [CrossRef]

10. Vega-López, S.; Venn, B.J.; Slavin, J.L. Relevance of the glycemic index and glycemic load for body weight, diabetes, and cardiovascular disease. Nutrients 2018, 10, 1361. [CrossRef] [PubMed]

11. Sacks, F.M.; Carey, V.J.; Anderson, C.A.; Miller, E.R., 3rd; Copeland, T.; Charleston, J.; Harshfield, B.J.; Laranjo, N.; McCarron, P.; Swain, J.; et al. Effects of high vs low glycemic index of dietary carbohydrate on cardiovascular disease risk factors and insulin sensitivity: The OmniCarb randomized clinical trial. JAMA 2014, 312, 2531-2541. [CrossRef] [PubMed]

12. Nazare, J.A.; De Rougemont, A.; Normand, S.; Sauvinet, V.; Sothier, M.; Vinoy, S.; Désage, M.; Laville, M. Effect of postprandial modulation of glucose availability: Short-and long-term analysis. Br. J. Nutr. 2010, 103, 1461-1470. [CrossRef] [PubMed]

13. Koloverou, E.; Esposito, K.; Giugliano, D.; Panagiotakos, D. The effect of Mediterranean diet on the development of type 2 diabetes mellitus: A meta-analysis of 10 prospective studies and 136,846 participants. Metabolism 2014, 63, 903-911. [CrossRef]

14. Esposito, K.; Maiorino, M.I.; Bellastella, G.; Chiodini, P.; Panagiotakos, D.; Giugliano, D. A journey into a Mediterranean diet and type 2 diabetes: A systematic review with meta-analyses. BMJ Open 2015, 5, e008222. [CrossRef] [PubMed]

15. Tuttolomondo, A.; Simonetta, I.; Daidone, M.; Mogavero, A.; Ortello, A.; Pinto, A. Metabolic and Vascular Effect of the Mediterranean Diet. Int. J. Mol. Sci. 2019, 20, 4716. [CrossRef]

16. Bergia, R.E.; Biskup, I.; Giacco, R.; Costabile, G.; Gray, S.; Wright, A.; Vitale, M.; Campbell, W.W.; Landberg, R.; Riccardi, G. The MEDGICarb-Study: Design of a multi-center randomized controlled trial to determine the differential health-promoting effects of low- and high-glycemic index Mediterranean-style eating patterns. Contemp. Clin. Trials Commun. 2020, 19, 100640. [CrossRef] 
17. Grundy, S.M.; Brewer, H.B.; Cleeman, J.I.; Smith, S.C.; Lenfant, C. Definition of metabolic syndrome: Report of the National Heart, Lung, and Blood Institute/American Heart Association conference on scientific issues related to definition. Circulation 2004, 109, 433-438. [CrossRef]

18. Brand-Miller, J.; Wolever, T.M.; Colagiuri, S.; Foster-Powell, K. The New Glucose Revolution: The Glycemic Index Solution for Optimum Health; Hodder, 2002; Available online: https:/ /books.google.com/books?id=noWOSQAACAAJ (accessed on 6 June 2021).

19. Giacco, R.; Costabile, G.; Della Pepa, G.; Anniballi, G.; Griffo, E.; Mangione, A.; Cipriano, P.; Viscovo, D.; Clemente, G.; Landberg, R.; et al. A whole-grain cereal-based diet lowers postprandial plasma insulin and triglyceride levels in individuals with metabolic syndrome. Nutr. Metab. Cardiovasc. Dis. 2014, 24, 837-844. [CrossRef]

20. Matthan, N.R.; Ausman, L.M.; Meng, H.; Tighiouart, H.; Lichtenstein, A.H. Estimating the reliability of glycemic index values and potential sources of methodological and biological variability. Am. J. Clin. Nutr. 2016, 104, 1004-1013. [CrossRef]

21. Little, R.J.A.; Rubin, D.B. Bayes and Multiple Imputation. In Statistical Analysis with Missing Data, 2nd ed.; John Wiley \& Sons, Inc.: Hoboken, NJ, USA, 2002; pp. 200-220.

22. Kolb, H.; Kempf, K.; Röhling, M.; Martin, S. Insulin: Too much of a good thing is bad. BMC Med. 2020, 18, 224. [CrossRef]

23. Rautio, N.; Jokelainen, J.; Oksa, H.; Saaristo, T.; Peltonen, M.; Puolijoki, H.; Tuomilehto, J.; Vanhala, M.; Moilanen, L.; Uusitupa, M.; et al. Changes in glucose metabolism in people with different glucose metabolism disorders at baseline: Follow-up results of a Finnish national diabetes prevention programme. Diabet Med. 2015, 32, 1611-1616. [CrossRef] [PubMed]

24. Selvin, E.; Steffes, M.W.; Zhu, H.; Matsushita, K.; Wagenknecht, L.; Pankow, J.; Coresh, J.; Brancati, F.L. Glycated hemoglobin, diabetes, and cardiovascular risk in nondiabetic adults. N. Engl. J. Med. 2010, 362, 800-811. [CrossRef] [PubMed]

25. Guess, N.D. Dietary interventions for the prevention of type 2 diabetes in high-risk groups: Current state of evidence and future research needs. Nutrients 2018, 10, 1245. [CrossRef] [PubMed]

26. Solomon, T.P.J.; Haus, J.M.; Kelly, K.R.; Cook, M.D.; Filion, J.; Rocco, M.; Kashyap, S.R.; Watanabe, R.M.; Barkoukis, H.; Kirwan, J.P. A low-glycemic index diet combined with exercise reduces insulin resistance, postprandial hyperinsulinemia, and glucosedependent insulinotropic polypeptide responses in obese, prediabetic humans. Am. J. Clin. Nutr. 2010, 92, 1359-1368. [CrossRef] [PubMed]

27. Brand-Miller, J.; Hayne, S.; Petocz, P.; Colagiuri, S. Low-glycemic index diets in the management of diabetes: A meta-analysis of randomized controlled trials. Diabetes Care 2003, 26, 2261-2267. [CrossRef] [PubMed]

28. Augustin, L.S.A.; Kendall, C.W.C.; Jenkins, D.J.A.; Willett, W.C.; Astrup, A.; Barclay, A.W.; Björck, I.; Brand-Miller, J.C.; Brighenti, F.; Buyken, A.E.; et al. Glycemic index, glycemic load and glycemic response: An International Scientific Consensus Summit from the International Carbohydrate Quality Consortium (ICQC). Nutr. Metab. Cardiovasc. Dis. 2015, 25, 795-815. [CrossRef]

29. Danne, T.; Nimri, R.; Battelino, T.; Bergenstal, R.M.; Close, K.L.; DeVries, J.H.; Garg, S.; Heinemann, L.; Hirsch, I.; Amiel, S.A.; et al. International consensus on use of continuous glucose monitoring. Diabetes Care 2017, 40, 1631-1640. [CrossRef]

30. Gorst, C.; Kwok, C.S.; Aslam, S.; Buchan, I.; Kontopantelis, E.; Myint, P.K.; Heatlie, G.; Loke, Y.; Rutter, M.K.; Mamas, M.A Long-term glycemic variability and risk of adverse outcomes: A systematic review and meta-analysis. Diabetes Care 2015, 38, 2354-2369. [CrossRef]

31. Lachin, J.M.; Bebu, I.; Bergenstal, R.M.; Pop-Busui, R.; Service, F.J.; Zinman, B.; Nathan, D.M. Association of glycemic variability in type 1 diabetes with progression of microvascular outcomes in the diabetes control and complications trial. Diabetes Care 2017, 40,777-783. [CrossRef]

32. Chakarova, N.; Dimova, R.; Grozeva, G.; Tankova, T. Assessment of glucose variability in subjects with prediabetes. Diabetes Res. Clin. Pract. 2019, 151, 56-64. [CrossRef]

33. Salas-Salvadó, J.; Bulló, M.; Babio, N.; Martínez-González, M.Á.; Ibarrola-Jurado, N.; Basora, J.; Estruch, R.; Covas, M.I.; Corella, D.; Arós, F.; et al. Reduction in the incidence of type 2 diabetes with the mediterranean diet: Results of the PREDIMED-Reus nutrition intervention randomized trial. Diabetes Care 2011, 34, 14-19. [CrossRef]

34. Schwingshackl, L.; Chaimani, A.; Hoffmann, G.; Schwedhelm, C.; Boeing, H. A network meta-analysis on the comparative efficacy of different dietary approaches on glycaemic control in patients with type 2 diabetes mellitus. Eur. J. Epidemiol. 2018, 33, 157-170. [CrossRef]

35. Bozzetto, L.; Alderisio, A.; Giorgini, M.; Barone, F.; Giacco, A.; Riccardi, G.; Rivellese, A.A.; Annuzzi, G. Extra-virgin olive oil reduces glycemic response to a high-glycemic index meal in patients with type 1 diabetes: A randomized controlled trial. Diabetes Care 2016, 39, 518-524. [CrossRef]

36. Dickinson, S.; Colagiuri, S.; Faramus, E.; Petocz, P.; Brand-Miller, J.C. Postprandial hyperglycemia and insulin sensitivity differ among lean young adults of different ethnicities. J. Nutr. 2002, 132, 2574-2579. [CrossRef] [PubMed]

37. Appel, L.J.; Moore, T.J.; Obarzanek, E.; Vollmer, W.M.; Svetkey, L.P.; Sacks, F.M.; Bray, G.A.; Vogt, T.M.; Cutler, J.A.; Windhauser M.M.; et al. A clinical trial of the effects of dietary patterns on blood pressure. DASH Collaborative Research Group. N. Engl. J. Med. 1997, 336, 1117-1124. [CrossRef] [PubMed]

38. Estruch, R.; Ros, E.; Salas-Salvado, J.; Covas, M.I.; Corella, D.; Aros, F.; Gomez-Gracia, E.; Ruiz-Gutierrez, V.; Fiol, M.; Lapetra, J.; et al. Primary prevention of cardiovascular disease with a Mediterranean diet supplemented with extra-virgin olive oil or nuts. N. Engl. J. Med. 2018, 21, 378.e34. [CrossRef]

39. Rodríguez-Rejón, A.I.; Castro-Quezada, I.; Ruano-Rodríguez, C.; Ruiz-López, M.D.; Sánchez-Villegas, A.; Toledo, E.; Artacho, R.; Estruch, R.; Salas-Salvadó, J.; Covas, M.I.; et al. Effect of a Mediterranean diet intervention on dietary glycemic load and dietary glycemic index: The PREDIMED study. J. Nutr. Metab. 2014, 2014, 985373. [CrossRef] [PubMed] 